
\title{
Research S Suare \\ Quality of Inpatient Care of Small and Sick Newborns in Pakistan: A Qualitative Study With Key Stakeholders
}

Nousheen Akber Pradhan ( $\nabla$ nousheen.pradhan@aku.edu )

Aga Khan University

Ammarah Ali

Aga Khan University

Sana Roujani

Aga Khan University

Sumera Aziz Ali

Columbia University

\section{Samia Rizwan}

United Nations International for Children's Education Fund, Country Office (UNICEF), Pakistan

\section{Sarah Saleem}

Aga Khan University

Sameen Siddiqi

Aga Khan University

\section{Research Article}

Keywords: Quality, newborns, health, stakeholders

Posted Date: February 16th, 2021

DOI: https://doi.org/10.21203/rs.3.rs-196477/v1

License: (c) (1) This work is licensed under a Creative Commons Attribution 4.0 International License.

Read Full License 


\section{Abstract \\ Background}

In LMICs including Pakistan, neonatal health and survival is a critical challenge, and therefore improving the quality of facility-based newborn care services is instrumental in averting newborn mortality. This paper presents the perceptions of the key stakeholders in the public sector to explore factors influencing the care of small and sick newborn and young infants in inpatient care settings across Pakistan.

\section{Methods}

This exploratory study was part of a larger study assessing the situation of newborn and young infant inpatient care provided across all four provinces and administrative regions of Pakistan. We conducted 43 interviews. Thirty interviews were conducted with the public sector health care providers involved in newborn and young infant care and 13 interviews were carried out with health planners and managers working at the provincial level. A semi-structured interview guide was used to explore participants' perspectives on enablers and barriers to the quality of care provided to small and sick newborns at the facility level. The interviews were manually analyzed using thematic content analysis.

\section{Findings:}

The study respondents identified multiple barriers contributing to the poor quality of small and sick newborn care at inpatient care settings. This includes an absence of neonatal care standards, inadequate infrastructure and equipment for the care of small and sick newborn, deficient workforce for neonatal case management, inadequate thermal care management for newborns, inadequate referral system, absence of multidisciplinary approach in neonatal case management and need to institute strong monitoring system to prevent neonatal deaths and stillbirths. The only potential enabling factor was the improved federal and provincial oversight for reproductive, maternal, and newborn care.

\section{Conclusion}

This qualitative study was insightful in identifying the challenges that influence the quality of inpatient care for small and sick newborn and the resources needed to fix these. There is a need to equip Sick Newborn Care Units with needed supplies, equipment and medicines, deployment of specialist staff, strengthening of in-service training and staff supervision, liaison with the neonatal experts in customizing neonatal care guidelines for inpatient care settings and to inculcate the culture for inter-disciplinary team meetings at inpatient care settings across the country.

\section{Introduction}


Facility-based newborn care refers to the specialized health care services provided by skilled health care personnel $24 / 7$ at the secondary and tertiary care units[1,2]. Improving the quality of inpatient care is instrumental in reducing neonatal mortality rate (NMR), particularly in low and middle-income countries (LMICs); which accounts for $98 \%$ of neonatal deaths[3, 4]. In pursuit of achieving the target of reducing neonatal mortality to 12 per 1000 live births of Sustainable Development Goal (SDG) 3 [5], it is imperative to improve the quality of inpatient care services. Also, stillbirths account for over half of the perinatal deaths, are often underreported, and remain an unprioritized agenda across the globe[6]. Despite slower progress in reducing neonatal mortality globally; facility-based neonatal care has remained underprioritized and underfunded, particularly in resource-constrained settings [7].

Globally, Pakistan has been reported to have the highest NMR and the country has been regarded as the riskiest place for a child to be born [8]. Federal and provincial departments of health have addressed neonatal care in Maternal, Newborn, and Child Health (MNCH) plans [9]. Also, UNICEF has supported the Government of Pakistan in establishing 38 Sick Newborn Care Units (SNCUs) at public health care facilities in the country[10]. Collaborative efforts have helped in declining NMR in the country from 55 deaths per 1,000 live births to 42 deaths per 1,000 live births during the last five years[11]. However, the number of newborn deaths is still unacceptably high.

While designing effective strategies to improve the quality of inpatient care for newborns, it is imperative to understand context-specific factors. Therefore, the closure of quality gaps in inpatient care services for small and sick newborns, among others, demands identifying the health system bottlenecks from stakeholders' (front line health care delivery staff, planners, and implementers' at the provincial level) perspectives to address the gaps in neonatal inpatients care. Strengthening of the existing facility-based system for the care of sick children is the most practical and cost-effective approach to avert preventable newborn deaths and is also central to achieving the goals of the Every Newborn Action Plan (ENAP)[12]. To date, newborn survival in Pakistan has received attention through various research and policy interventions. The research in this area is mainly clustered around establishing step down units at the facility for low birth weight babies [13], economic evaluations to measure maternal and neonatal health $(\mathrm{MNH})$ service costs [14], assessment of service readiness in offering $\mathrm{MNH}$ services at facility level[15], assessing the outcomes for neonatal care admissions [16] to health workers' competency assessment on $\mathrm{MNH}$ aspects in the country [17]. Besides this, integration of neonatal health into the existing national and provincial $\mathrm{MNCH}$ program[18], and inclusion of neonatal component in integrated management of neonatal and childhood illnesses strategy, since 2000 are some of the policy level initiatives[19].

Exploring perspectives of the front line care providers of neonatal care and health sector planners and managers has received less attention in unfolding the bottlenecks at the health systems level. This paper, therefore, presents findings from the qualitative study with the key stakeholders to explore their perceptions regarding the factors influencing the quality of care for small and sick newborns at inpatient care facilities in Pakistan.

\section{Methods}


This qualitative exploratory study is part of a larger cross-sectional study to assess the readiness and quality of inpatient care for NYIs provided in public sector hospitals across Pakistan[20]. The data for the study was gathered from a sample of public sector healthcare facilities, spread across all the provinces and regions of the country, providing inpatient care for newborns and young children (0-59 days of age). Out of 38 UNICEF supported hospitals across Pakistan, 23 were purposively selected in consultation with UNICEF country office, and the Ministry of National Health Services Regulation \& Coordination. These included 3 tertiary care hospitals, 6 provincial/regional referral hospitals, and 14 District Headquarter (DHQ) Hospitals. And, offices of the Director-General (DG) Health across four provinces and two federal territories.

Key informant interviews were conducted with two groups of respondents; (1) healthcare providers $(n=30)$ directly or indirectly involved in the care of newborn and young infants including in-charge of health facilities (Medical Superintendent-MS) or their representatives, and service managers with responsibilities related to newborn and young infant (NYIs) care. And (2) managers and planners $(n=13)$ at the district and provincial level including DG Health Services, MNCH program Director, and MNCH Program Managers at the Department of Health in all four provinces of the country. Up to five respondents per facility (inclusive of the medical and nursing workforce) were interviewed. Where there was a choice, the most experienced person in providing the neonatal care service, present at the time of the assessment was interviewed. The average duration of interviews was 45-60 min.

The interviews were conducted using a semi-structured interview guide to study barriers and enablers for inpatient care of small and sick newborns in Pakistan. The questions focused on the quality of inpatient care for small and sick newborns at the respective facilities, availability of infrastructure support, availability and expertise of specialized staff, and training of staff in neonatal care. The special emphasis was given to the associated challenges in the existing health care system in the provision of neonatal care at the facilities. This paper was not looking at the strategies to address implementation issues related to NYI care in inpatient care settings. The solutions to implementation bottlenecks are addressed in the main paper.

All the interviews were held during April-May 2019 and were conducted in dual languages i.e., Urdu and English. The data gathered through interviews in the local language was transcribed and translated into English. Transcripts were proofread multiple times to get the correct understanding of the information. The transcripts were manually analyzed using Graneheim \& Landman, 2004 content analysis approach [21]. The main ideas on the transcripts were selected and labeled as "codes". Similar codes were categorized and assembled into categories. In the final step, sub-categories with similar concepts were grouped into "themes" (Table 1). Meetings were held among the team members to mutually agree on the codes, categories, and themes derived from the qualitative data set. All study participants were interviewed after explaining the purpose of the study and obtaining written informed consent. The confidentiality of the participants was maintained by assigning a unique code to safeguard their identity. Prior ethical clearance was obtained from the Ethics Review Committee of the Aga Khan University, Pakistan, and the National Bioethics Committee, Pakistan. 


\section{Findings}

Findings obtained from the interviews are grouped under two broad themes; (1) Barriers contributing to the poor quality of inpatient care for small and sick newborn care. This includes perceived bottlenecks and challenges impeding the quality of small and sick newborn care, and (2) Enablers contributing to improved inpatient care for small and sick newborns. This theme identified one factor potentially contributing to improved care for small and sick newborns. These two themes are explained in the following sections.

\section{Theme l: Barriers contributing to the poor quality of inpatient care for small and sick newborns across Pakistan}

\section{Category 1: Lack of essential neonatal care standards}

Both groups of respondents (health care providers and planners) expressed their dissatisfaction over the quality of care provided to small and sick newborns at the district and tertiary care units across Pakistan. Among the underlying reasons, the non-availability and use of essential newborn care guidelines/standards at the facility by front line service delivery staff were highlighted as the major concern. A group of pediatricians and senior representatives at the $\mathrm{MNCH}$ Provincial Directorate voiced their concerns by highlighting the non-availability of standards protocols for preventing intrapartum stillbirths and neonatal deaths and common childhood illnesses and recommended for the development of these standards at the provincial and district level.

"There are no protocols. No standard protocols are being followed for newborn and neonatal care." (MNCH Assistant Director, 005-01)

"They (district and the provincial department of health officials) should make proper guidelines for the management of major issues such as birth asphyxia, preterm infections, etc." (Pediatrician, 003-05)

A respondent while reflecting the current practice of keeping 3-4 neonates in a single cot, stated;

"Now high standard means one child per bed. In that room (nursery), there are four newborns in one bed. So, what kind of standard we are fulfilling?" (Pediatrician, 001-02)

An obstetrician expressed concerns that the absence of set guidelines encouraged malpractices related to the care of a newborn;

"There is no newborn care as such. An obstetrician will try to save the woman during delivery and will hand over the baby to TBA (Dai/ Aya), and they will turn the baby upside down and slap her to make her cry. No vitals, no APGAR score, no explanation regarding the danger signs is carried out. Even at the tertiary level, the system is not up to the mark. So, what can you expect from Rural Health Center, Basic Health Units, and DHQs?" (MS, 001-03) 
While respondents shared their grievances about the absence of neonatal care standards, they also acknowledged World Health Organization (WHO) protocols of essential maternal and neonatal care and highlighted the need for implementing the same at SNCUs.

\section{Category 2: Inadequate infrastructure and equipment for the care of small and sick newborn}

Health care providers including the MS, obstetricians/ gynecologists, and pediatricians also expressed concerns about the inadequacy of infrastructure and equipment in health facilities as an important reason for the poor quality of neonatal care. Infrastructural deficiencies included unavailability or nonfunctionality of neonatal intensive care units (NICU), labor room, ventilators, life-saving medications, shortage of the required number of infant cots and ultrasound facility, non-functional operation theaters, etc. The resulting poor quality of care contributed to neonatal deaths at these facilities.

"There are a lot of neonatal deaths in the facility as well. The reasons are that we have a shortage of manpower, the need for electricity is not fulfilled. If the incubator is required, it's not provided ........there are few medicines........these are the problems due to which neonatal deaths are reported". (Obstetrician, 001-04)

"A preterm baby, less than 28 weeks needs ventilator support, which we are unable to provide. So, what do you expect? How can we make progress in this situation?" (Gynecologist, 002-03)

Frontline care providers believed that an early discharge of preterm neonates is due to limited bed capacity at the DHQ hospitals.

"One issue is the unavailability of space, because of which at times we are compelled to discharge preterm neonates (Gynecologist, 002-03)

Furthermore, the pediatricians also shared their views about the compromised quality of care in the absence of required logistics and essential lifesaving medications for sick newborn;

“..........If a child is presented with fits and I don't have valium or when newborn is not breathing, I don't have an ambu bag to resuscitate, the baby will die. This equipment should be made available". (Pediatrician, 007-09)

Furthermore, both groups of respondents also highlighted the scarcity of incubators and ventilators in rural areas.

"After delivery, if the baby gets hypothermia, fever or asphyxia then there is no neonatal set up in the rural areas to manage such cases. Our ambulances are not equipped with ventilator services. They do not have the incubator or the warmer..." (MNCH Assistant Director, 005-01)

Moreover, some of the respondents at the facility level also shared their grievances about the inadequacy of oxygen supply and related equipment (ambulatory bags, oxygen flow meters, etc.). 


\section{Category 3: Issues with neonatal health care workforce}

\section{Sub-category 3.1: Deficiency of specialized workforce for neonatal case management}

Inadequate availability of the specialized workforce for neonatal case management was perceived as another hurdle in delivering optimum quality of care. Both groups of respondents equally identified the shortage of neonatologists, pediatricians, and nurses in the neonatal units.

A pediatrician while highlighting the dearth of required workforce exclaimed;

".......We do not have a single neonatologist in the entire province. Pediatricians deal with neonates. They are not present in every DHQ. The staff is not enough across different units of the hospital...." (Paediatrician, 007-04)

Respondents while reflecting the unavailability of a specialized workforce round the clock at the inpatient care units, shared that evening and night shifts are mostly observed without nurses. Alongside the unavailability of nurses, pediatricians were reported to be on call and were only reported to be accessible in the daytime; resulting in the delay of essential newborn care.

"The patient care is affected because of the shortage of staff in shifts. Let's suppose cesarean has been done and now they need a child specialist, but the child specialist is on call.... During this calling process, the golden minutes are wasted. And when the pediatrician arrives, the baby has died already, or he has been resuscitated." (MNCH Assistant Director, 005-01)

Dialogues with the $\mathrm{MNCH}$ program planners and managers unfolded the reasons contributing to the dearth of the specialized workforce at inpatient care units. A commonly reported reason was the budgetary constraints at the provincial level; restricting recruitment of skilled workforce. While highlighting the inadequacy of the specialized neonatal care workforce across DHQs, suggestions were proposed for in-service training of residents in neonatology.

"There should be more people sent for the capacity building. Those who have done house jobs in the pediatrics should be sent to neonatology units. They should learn and build their capacity and then get appointed in the districts." (DG Health, 007-02)

\section{Sub-category: 3.2. Inadequate thermal care management for newborns}

While respondents highlighted multiple aspects of poor quality of newborn care at the facility level, it came under discussion from one province that newborns are handled by the traditional birth attendants (TBAs); resulting in inadequate thermal care, which leads to neonatal deaths at the facility.

"....... So, what l've observed is that the child is handed over to the TBA, she takes the child somewhere and doesn't keep him warm.... They just dry the child with a cloth and the child remains cold later. A lot of children die because they remain cold." (Pediatrician,001-01) 
One of the senior officials at the Provincial Department of Health also shared similar malpractice after childbirth.

"Soon after the delivery, if the male baby is born, he is handed over to ward servants who then handover him to family. They wander all-around taking the baby thus causing hypothermia. This has been strictly mentioned a lot of times that baby should be placed in front of the heater or the baby warmer should be switched on at least half an hour before the delivery."

(DG Health, 001-01)

\section{Category 4: Inadequate referral system}

Across the regions, the inadequate referral system in general and in particular to neonatal cases was repeatedly emphasized. While unfolding the issues in the referral system, participants reported a lack of pre-referral care and treatment by attending physicians reflecting incompetency of front-line staff in managing emergency cases requiring urgent referrals.

"..... The doctors are incapable of resuscitation and proper referrals, this leads to a situation where a critically ill patient arrives with no proper referral or support, so we must send him forward." (DG Health, 001-01)

Another official in the Department of Heath straightforwardly ascribed stillbirths and neonatal deaths in the country to a poor referral system.

"One of the challenges in preventing stillbirths and neonatal deaths in the poor referral system and poor transportation." (MNCH Coordinator, 004-01)

In the views of pediatricians, the absence of follow-up after the referral was also highlighted, especially when the sick child is referred from peripheries to the tertiary care hospitals.

"We don't have a system of follow-up after referral at all. When a child from a small village is referred to a hospital in the city, there is no follow-up; whether the child was given the service or not, whether the ambulance was arranged on their own or by the government or by some NGO, we do not have such system right now." (Pediatrician, 003-01)

While the existence of emergency transportation arrangements for maternal and sick newborn cases in the form of emergency helpline numbers was acknowledged by $\mathrm{MNCH}$ managers and planners. However, the non-availability of ambulance service for patients in general and particularly related to sick newborns remain cited as a concern.

\section{Category 5: Absence of a multidisciplinary approach in neonatal case management}

Narratives from stakeholders' interviews also alluded to the lack of coordination mechanism among three different specialties i. e., neonatology, pediatrics, and gynecology in inpatient care units. During 
emergency events such as fetal distress, the absence of a neonatologist exhibited a lack of coordination among attending staff, which can risk newborn life.

"Now the protocol in the case of fetal distress is that when an obstetrician is in OT to deliver the baby, there should be a neonatologist present there too. We call them... but they do not reach on time."

(Gynecologist, 006-01)

The underlying reasons that surfaced for the lack of coordination between the specialist cadres were indicative of wrongly perceived role and responsibilities by the respondents;

"A Gynecologist is more interested in maternal health and stillbirths; whereas a pediatrician is only interested in neonatal deaths. There is a lack of coordination between these two cadres." (MNCH Coordinator, 004-01)

The respondents while sharing their concerns about the lack of coordination between the diverse group of providers rebuked the hospital governance, and strongly emphasized the culture of teamwork while providing care for sick neonates;

".....There are communication gaps between the departments...... It is not only the administration or the government who is responsible. Responsibility, of course, lies upon the gynecologists, obstetricians, and pediatricians as well. We should build an environment where there is teamwork." (Gynecologist, 006-01)

\section{Category 6: Need to institute monitoring system to prevent neonatal deaths and stillbirths}

Alongside the above-mentioned issues associated with the poor quality of neonatal care at the facility level, respondents also discussed instituting and strengthening monitoring systems. This includes careful reviews of patient case records, verbal autopsies, perinatology meetings, etc. While highlighting the need for reviewing the patient reports and undertaking verbal autopsies for neonatal deaths and stillbirths, a pediatrician stated;

"Reviewing of reports can give us data, for example, if we report 10 deaths in a month, then we can review to see what were the causes and check if it was a mistake on our part or from the parents' side so that we can prevent it from happening again and we can save child's life." (Pediatrician, 003-01)

Participants indicated a culture where facility-based supervision is nearly non-existent. In this regard, pediatricians suggested monthly audits by the provincial health department representative and instituting a formal mechanism at the facility and district level to ascertain the causes of neonatal deaths.

"Formal mechanisms with standardized forms can help identify causes and prevent stillbirths and neonatal deaths. If we recommend these forms to the government, then it will get you data about the causes of stillbirths and neonatal deaths." (Pediatrician, 001-02)

Furthermore, a few of the respondents also highlighted the need for supervisory visits (audits) by the respective provincial health department to ensure correct utilization of the logistic support received from 
UNICEF at SNCUs.

"Whichever hospital we are working in, whatever equipment we are getting from UNICEF etc. or the training received, there should be a follow-up to see if all of that is provided is being utilized. We don't have a system for audits." (Pediatrician, 003-01)

\section{Theme Il: Enablers contributing to improved inpatient care for small sick newborns}

\section{Category 1: Improved federal and provincial oversight for reproductive, maternal, and newborn (RMNCH) care}

The existence of the RMNCH technical group at the federal and provincial levels was positively viewed by the stakeholders with the potential to improve reproductive, maternal, and newborn care. The respondents viewed that the technical committee is actively working to improve the $\mathrm{MNCH}$ across Pakistan. The group comprised of government, non-government, and International Non-Government Organizations (INGOs) with the representation of Health Secretaries, Director General Health (National and Provincial), planning \& finance representatives, WHO, and UNICEF. Health care professionals including obstetricians and pediatricians are also reported to be part of the said group. The committee is to promote evidence-based $\mathrm{MNCH}$ interventions across the country, alongside advocacy for essential newborn interventions such as umbilical cord care by chlorhexidine at the facility level.

\section{Discussion}

This exploratory study illustrates the compromised quality of care for small and sick newborns at inpatient care units across hospitals in Pakistan. The findings obtained in this study are part of a larger study with a systematic assessment of 23 SNCUs across the country. The findings substantiate concerns expressed by the very stakeholders who are responsible for providing and managing neonatal care of the dismal state of public sector hospitals that is a key contributor to the high neonatal mortality in the country.

Poor quality of facility-based care for the small and sick newborn is a major impediment towards the attainment of universal health coverage and SDG-3. A 12 country study confirmed our results that the interventions with the most perceived bottlenecks are facility-based where rapid emergency care is needed, notably inpatient care of small and sick newborns, treatment of neonatal infections, and KMC [22]. Equally important is to ensure the integration of essential newborn care into countries' policies and programs [23]. In wake of high neonatal mortality in the country, the availability of essential newborn care standards and guidelines for inpatient care units are essential, which many LMICs lack while offering care to newborns $[22,23]$. Similar findings were obtained in our study regarding the absence of standards and guidelines for neonatal care. The unavailability of neonatal care standards at inpatient care units across the districts reflects that the government's commitment to improving neonatal health, detailed in $\mathrm{MNCH}$ action plans [9] has not translated into actions at the facility level. Studies indicate that countries with high maternal, perinatal, and neonatal mortality have an inadequate and poor quality of health care 
services and, thus increased emphasis is placed on the need for the standards of care[24]. WHO has a good stock of newborn care standards which can be customized and made available for service providers at the inpatient care units $[24,25]$ in Pakistan.

Ensuring optimum care for small and sick newborns demands adequate infrastructure support with required supplies, equipment, and stock of essential drugs at NICUs. Unfortunately, among the underlying reasons that surfaced for the poor quality of neonatal care were inclusive of inadequate neonatal care infrastructure at DHQs across the country. These findings also correspond with studies in Kenya[26] and Bangladesh[27], exhibiting poor structural support for neonatal care. Health care providers in Bangladesh reported inadequacy of essential logistic support, such as non-functional incubators, alongside the unavailability of the required instruments and reagents to undertake laboratory tests [27]. Our study participants highlighted limited bed capacity across DHQs; resulting in an early discharge of pre-term neonates. Likewise, findings obtained from the multi-country analysis from five out of 12 countries (from Asian and African regions), also documented limited space for sick newborns and for mothers at inpatient care units [7]. Furthermore, poor functionality of neonatal care equipment was also documented from India, with a lack of power backups for SNCUs[28], as was the case in our study. Under essential newborn care, neonatal resuscitation is among the essential component needed for the care of small and sick neonates at inpatient care units. Concerns were raised about the inadequacy of oxygen supplies and equipment at SNCUs. Likewise, findings across LMICs have also demonstrated similar hurdles for neonatal resuscitation, with equipment and supplies such as bags and masks not readily available at the facility level [29].

Furthermore, the stock of a specialized health workforce is essential to ensure quality assured neonatal care services at inpatient care units. Unfortunately, alongside other bottlenecks in neonatal care service delivery at SNCUs, this study also informed about the critical health workforce gap in various cadres; pediatrics, neonatology, and nursing. Unavailability of pediatricians after cesarean section and their delayed arrival has been reported to be associated with wastage of golden hour in this study. The "Golden hour" concept includes practicing all the evidence-based interventions for neonates in the initial sixty minutes for better long-term outcomes [30]. The shortage of the required health workforce has been perceived as a major obstacle affecting NICUs [31]. Likewise, an inadequate specialized workforce in numbers and skills were also documented from other countries [7].

Our findings also reveal that essential newborn care aspects are not up to the required standards; presumably due to the absence of newborn care standards and poor monitoring at inpatient care facilities. As a result, neonatal deaths are being reported at the facility. This urgently calls for staff training in essential newborn care with a focus on thermal care management. Thermal protection and care of newborns are highly recommended, as it reduces hypothermia; known to contribute to global neonatal mortality and comorbidity of other major causes of neonatal death [32]. The WHO practical guide for the thermal protection of the newborn recommends several key interventions to ensure that the newborn is kept warm. This includes that the place of delivery is warm, newborns are immediately dried and either wrapped or placed on the mother for skin-to-skin contact [33]. Consistent with earlier studies in 
Nepal [34] and Tanzania [35], our study also reports sub-optimal thermal care management for newborn care by unskilled staff.

Furthermore, a multidisciplinary approach has been highly recommended in neonatal care management, as it leads to integrated care and also facilitates developing rapport with parents [30,36]. It is not uncommon to note that shortcomings in teamwork for neonatal case management have been reported from earlier studies [37], and our study findings also highlighted the absence of coordination among the care providers from varied disciplines. The underlying reasons could be attributed to the absence of a neonatal care protocol with a mandatory requirement for a multidisciplinary approach in neonatal care, and the lack of accountability mechanism at inpatient care units. Moreover, this study also reaffirmed findings from earlier studies about the inadequate monitoring of sick neonates, with particular attention on instituting a mechanism of perinatal death audits [28, 38].

The barriers identified in the study strongly calls to review the accountability mechanism at all three levels; (1) facility level, (2) district health authority, and (3) provincial health departments and clarifying their roles and responsibilities in overcoming the barriers in the provision of care of small and sick newborns. While provincial health departments are responsible to oversee the provision of health care services and liaison with district health offices to provide support to districts. On the other hand, district health offices are mandated to provide needed services in their districts through designated public health care facilities. Facility administration is the third tier with responsibility delegated to deliver and manage health care services. Identification of challenges in inpatient care services to small and sick newborn, therefore, calls for consultative meetings between the facility administrations, district health authorities, supported by the provincial $\mathrm{MNCH}$ steering committees to help districts and facility managers develop action plans towards closing the quality gap to improve NYls care in inpatient care settings. The provincial health department is responsible to resolve financial constraints related to the recruitment of specialized staff at the district level and also to build the capacity of district health authorities across various spheres in delivering optimum care to small and sick newborns at SNCs.

The study has strengths and limitations. To our knowledge, this is the first study at the national level which has not only captured insights of the front-line care providers deployed at SNCUs but also explored perspectives of planners and managers involved in the $\mathrm{MNCH}$ program on the quality of inpatient care at DHQs in the country. As the scope of the study limits to the public health care facilities, therefore perspectives from the care providers at private care hospitals were not included.

\section{Conclusions}

This qualitative study was insightful in identifying the challenges that influence the quality of inpatient care for small and sick newborns and the resources needed to fix these. Unless these challenges are addressed, neonatal mortality is unlikely to meaningfully decrease in Pakistan soon. The country that has emerged as one of the worst neonatal mortality indicators on the globe essentially needs to strengthen the quality of neonatal health service delivery by providing an enabling environment for improved 
newborn survival at the facility level. In this regard five actions are recommended at the facility and district level; (1) Strengthen SNCUs by providing needed equipment, supplies, and medicines, (2) Recruitment and deployment of Neonatologists, Neonatal Surgeons, Neonatal Nurses in district hospitals, (2) $\mathrm{MNCH}$ steering and coordination committee to liaise with the national and international experts in customizing neonatal care guidelines for inpatient care settings, (3) Strengthen pre-service and in-service training programs for health care providers across inpatient care settings in neonatal care management, and (4) $\mathrm{MNCH}$ steering and coordination committee to conduct monthly audits at inpatient care facilities to supervise staff performance and ensure perinatal and neonatal death reviews. Furthermore, (5) ensuring coordinated involvement of required specialists in offering care to sick neonates.

\section{List Of Abbreviations}

Aga Khan University AKU

Appearance, Pulse, Grimace, Activity, and Respiration APGAR

Director General DG

District Headquarter Hospital DHQ

Every Newborn Action Plan ENAP

International Non-Government Organizations INGOs

Low and Middle-Income Countries LMICs

Maternal and Neonatal Health MNH

Maternal, Newborn, and Child Health $\mathrm{MNCH}$

Medical Superintendent MS

Newborn Intensive Care Units NICUs

Newborn and Young Infants NYls

Neonatal Mortality Rate NMR

Reproductive, Maternal and Child Healthcare RMNCH

Sustainable Development Goal SDG

Sick Newborn Care Units SNCUs

United Nations International for Children's Education Fund, Country Office,Pakistan UNICEF 


\section{Declarations}

\section{Ethics approval and consent to participate}

Ethical clearance was obtained from AKU's Ethics Review Committee, as well as the National Bioethics Committee. Ethical considerations were duly taken care of during the training and conduct of the study. All respondents were approached for their informed verbal and written consent prior to data collection. Confidentiality of the information was maintained throughout and data was saved in a secure manner. All methods were carried out in accordance with relevant guidelines and regulations.

\section{Consent for publication}

Not applicable.

\section{Availability of data and materials}

The datasets used and/or analyzed during the current study are available from the corresponding author on reasonable request.

\section{Competing interests}

The authors declare that they have no competing interests.

\section{Funding}

The UNICEF has funded the study.

\section{Authors' contributions}

\section{Acknowledgements}

We are highly grateful to the support extended in the conduct of the study from the Ministry of National Health Services, Regulation and Coordination, Islamabad Pakistan and all Provincial and District Health Departments.

\section{Authors' information}

\section{Affiliations}

\section{Department of Community Health Sciences, Aga Khan University, Pakistan}

Ms. Nousheen Akber Pradhan; BScN; MSc

Dr. Sana Roujani; BDS; MSc 
Dr. Ammarah Ali; BDS; MSc

Dr. Sarah Saleem; MBBS; DCH, MSc, FCPS

Dr. Sameen Siddiqi; MBBS; MSc; FCPS; FFPH; DrMed

Department of Epidemiology, Columbia University New York, USA

Dr. Sumera Aziz Ali; MBBS; MSc; FCPS; PhD Scholar

\section{United Nations International for Children's Education Fund}

Dr. Samia Rizwan; MBBS; FCPS

\section{Authors' Contributions}

S.A. designed the study with technical input from S.S., Sa.S., S.R., and N.P. S.A. and N.P. executed the study, collected data and provided field supervision. N.P. developed the data collection tool and drafted the manuscript. A.A and Sa.R. provided support in the data collection, data analysis, and in manuscript writing. S.S. and Sa.S reviewed multiple drafts of the manuscript. All authors have read and approved the manuscript.

\section{References}

1. Darmstadt GL, Bhutta ZA, Cousens S, Adam T, Walker N, De Bernis L, et al. Evidence-based, costeffective interventions: how many newborn babies can we save? The Lancet. 2005;365(9463):97788.

2. Neogi SB, Malhotra S, Zodpey S, Mohan P. Does facility-based newborn care improve neonatal outcomes? A review of evidence. Indian pediatrics. 2012;49(8):651-8.

3. Lawn JE, Blencowe H, Oza S, You D, Lee AC, Waiswa P, et al. Every Newborn: progress, priorities, and potential beyond survival. The Lancet. 2014;384(9938):189-205.

4. Wang H, Liddell CA, Coates MM, Mooney MD, Levitz CE, Schumacher AE, et al. Global, regional, and national levels of neonatal, infant, and under-5 mortality during 1990-2013: a systematic analysis for the Global Burden of Disease Study 2013. The Lancet. 2014;384(9947):957-79.

5. UNICEF (2015). Advancing WASH in Schools Monitoring. Available at http://www.unicef.org/wash/schools/files/Advancing_WASH_in_Schools_Monitoring(1).pdf.

6. Reinebrant H, Leisher S, Coory M, Henry S, Wojcieszek A, Gardener G, et al. Making stillbirths visible: a systematic review of globally reported causes of stillbirth. BJOG: An International Journal of Obstetrics \& Gynaecology. 2018;125(2):212-24.

7. Moxon SG, Lawn JE, Dickson KE, Simen-Kapeu A, Gupta G, Deorari A, et al. Inpatient care of small and sick newborns: a multi-country analysis of health system bottlenecks and potential solutions. 
BMC pregnancy and childbirth. 2015;15(2):S7.

8. UNICEF. Every Child Alive. The urgent need to end newborn deaths . 2018. .

9. Pakistan MoHGo. National Maternal Newborn and Child Health (MNCH) Program. Pakistan2006.

10. Care for the preterm babies is imperative to improve child survival indicators in Pakistan [Available from: https://www.unicef.org/pakistan/press-releases/care-preterm-babies-imperative-improve-childsurvival-indicators-pakistan.

11. National Institute of Population Studies (NIPS) [Pakistan] and ICF. 2018. Pakistan Demographic and Health Survey 2017-18. Islamabad, Pakistan, and Rockville, Maryland, USA: NIPS and ICF. .

12. Organization WH. Every newborn: an action plan to end preventable deaths. 2014.

13. Bhutta ZA, Khan I, Salat S, Raza F, Ara H. Reducing length of stay in hospital for very low birthweight infants by involving mothers in a stepdown unit: an experience from Karachi (Pakistan). bmj. 2004;329(7475):1151-5.

14. Khowaja AR, Mitton C, Qureshi R, Bryan S, Magee LA, von Dadelszen P, et al. A comparison of maternal and newborn health services costs in Sindh Pakistan. PloS one. 2018;13(12).

15. Zaidi S, Riaz A, Rabbani F, Azam SI, Imran SN, Pradhan NA, et al. Can contracted out health facilities improve access, equity, and quality of maternal and newborn health services? Evidence from Pakistan. Health research policy and systems. 2015;13(1):S54.

16. Ali SR, Ahmed S, Lohana H. Disease patterns and outcomes of neonatal admissions at a secondary care hospital in Pakistan. Sultan Qaboos University medical journal. 2013;13(3):424.

17. Ariff S, Soofi SB, Sadiq K, Feroze AB, Khan S, Jafarey SN, et al. Evaluation of health workforce competence in maternal and neonatal issues in public health sector of Pakistan: an assessment of their training needs. BMC health services research. 2010;10(1):319.

18. National Maternal Neonatal and Child Health Program. Ministry of Health, Government of Pakistan [Available from: http://dynasoft.org/mnch/.

19. WHO. Child Health and Development. Implementation of IMCI in Pakistan [Available from: http://www.emro.who.int/child-health/strategy-implementation/implementation-of-imci-inpakistan.html.

20. Sumera Aziz NAP, Sana Roujani, Ammarah Ali, Zahid Abbsai, Shujaat Hussain, Sarah Saleem and Sameen Siddiqi. A Situational Analysis on Stillbirths, Newborn Deaths and Small and Sick Newborn Care Findings from Pakistan - 201. Department of Community Health Sciences, Aga Khan University, UNICEF and Ministry of National Health Services Regulation and Coordination, Islamabad, Pakistan; 2019.

21. Graneheim UH, Lundman B. Qualitative content analysis in nursing research: concepts, procedures and measures to achieve trustworthiness. Nurse education today. 2004;24(2):105-12.

22. Dickson KE, Kinney MV, Moxon SG, Ashton J, Zaka N, Simen-Kapeu A, et al. Scaling up quality care for mothers and newborns around the time of birth: an overview of methods and analyses of intervention-specific bottlenecks and solutions. BMC pregnancy and childbirth. 2015;15(S2):S1. 
23. Daly P, Taylor M, Tinker A. Integrating essential newborn care into countries' policies and programs. Population Reference Bureau, Washington DC. 2003.

24. Integrated Management of Pregnancy and Childbirth. Standards for Maternal and Neonatal Care.: WHO; 2007.

25. Organization. WH. Pregnancy, Childbirth, Postpartum and Newborn Care: A guide for essential practice Third Edition. 2015.

26. Murphy GA, Gathara D, Abuya N, Mwachiro J, Ochola S, Ayisi R, et al. What capacity exists to provide essential inpatient care to small and sick newborns in a high mortality urban setting?-A crosssectional study in Nairobi City County, Kenya. PLoS One. 2018;13(4):e0196585.

27. Islam F, Rahman A, Halim A, Eriksson C, Rahman F, Dalal K. Perceptions of health care providers and patients on quality of care in maternal and neonatal health in fourteen Bangladesh government healthcare facilities: a mixed-method study. BMC health services research. 2015;15(1):237.

28. Neogi S, Khanna R, Chauhan M, Sharma J, Gupta G, Srivastava R, et al. Inpatient care of small and sick newborns in healthcare facilities. Journal of Perinatology. 2016;36(3):S18-S23.

29. Enweronu-Laryea C, Dickson KE, Moxon SG, Simen-Kapeu A, Nyange C, Niermeyer S, et al. Basic newborn care and neonatal resuscitation: a multi-country analysis of health system bottlenecks and potential solutions. BMC pregnancy and childbirth. 2015;15(2):S4.

30. Sharma D. Golden hour of neonatal life: Need of the hour. Maternal health, neonatology and perinatology. 2017;3(1):16.

31. Toren $\mathrm{O}$, Nirel $\mathrm{N}$, Tsur $\mathrm{Y}$, Lipschuetz M, Toker A. Examining professional boundaries between nurses and physicians in neonatal intensive care units. Israel Journal of Health Policy Research. 2014;3(1):43.

32. Lunze K, Bloom DE, Jamison DT, Hamer DH. The global burden of neonatal hypothermia: systematic review of a major challenge for newborn survival. BMC medicine. 2013;11(1):24.

33. Organization WH. Thermal protection of the newborn: a practical guide. World Health Organization; 1997.

34. Falle TY, Mullany LC, Thatte N, Khatry SK, LeClerq SC, Darmstadt GL, et al. Potential role of traditional birth attendants in neonatal healthcare in rural southern Nepal. Journal of health, population, and nutrition. 2009;27(1):53-61.

35. Shamba D, Schellenberg J, Hildon ZJ-L, Mashasi I, Penfold S, Tanner M, et al. Thermal care for newborn babies in rural southern Tanzania: a mixed-method study of barriers, facilitators and potential for behaviour change. BMC pregnancy and childbirth. 2014;14:267-.

36. Barbosa VM. Teamwork in the neonatal intensive care unit. Physical \& occupational therapy in pediatrics. 2013;33(1):5-26.

37. Thomas E, Sexton J, Lasky R, Helmreich R, Crandell D, Tyson J. Teamwork and quality during neonatal care in the delivery room. Journal of Perinatology. 2006;26(3):163-9. 
38. Nakibuuka VK, Okong P, Waiswa P, Byaruhanga RN. Perinatal death audits in a peri-urban hospital in Kampala, Uganda. African health sciences. 2012;12(4):435-42.

\section{Tables}

Table 1. Findings from the in-depth interviews of the key stakeholders

Theme I: Barriers contributing to the poor quality of inpatient care for small and sick newborns across Pakistan 


\section{S.\# Categories}

1. Lack of essential neonatal care standards

1.

2. Inadequate infrastructure and equipment for the care of

newborn and sick infants

Issues with neonatal health care workforce

3.1. Deficiency of specialized workforce for neonatal case management

\section{Codes}

Protocol should be available

Unavailability of newborn care guidelines

Make proper guidelines

One child per bed

System of protocols

Criteria

Shortage of manpower

No electricity

Unavailability of NICUs

Unavailability of labor rooms

Unavailability of space

Staff not enough

Absence of baby cots

OT not working

Ultrasound facility

Early discharging preterm

No ambulatory bags

No Incubators

Single neonatologist

Cannot find pediatricians

staff is not enough

Neonatologist not available

one nurse in evening and night shift

Staff shortage

Golden minutes wasted

Child specialist on call

3.2 Inadequate thermal care management for newborns

Child still cold 
Hypothermia

Newborn dying

Baby handed over to TBAs

Lack of thermal care

Doesn't keep the baby warm

4.

Inadequate referral system

No proper referral or support

Must send him (patient)

forward

Unavailability of

transportation

No follow up after referral

Delay at DHQ level

Effective communication in emergency situations

5. Absence of multidisciplinary approach in neonatal case management

Neonatologist don't reach on time

Pediatrician interested in NNDs only

Communication gap

Lack of coordination

Need for teamwork

6. Need to institute strong monitoring system to prevent neonatal deaths and stillbirths

Review of reports

10 deaths in one months

Verbal autopsies

Cause of stillbirths and neonatal deaths

Audits and follow ups

Training needs

No audits

\section{Theme Il: Enablers contributing to improved inpatient care for small sick newborns}

1. Improved federal and provincial oversight for reproductive, Technical group maternal, and newborn (RMNCH) care

Actively working

WHO, UNICEF 


\section{INGOS}

\section{Promoting RMNCH care}

Evidenced based interventions

Umbilical cord care

This table presents the themes, categories and codes arriving from the in-depth interviews of the key stakeholders to assess their perspectives of the quality of in-patient care for small and sick newborns across public sector health care facilities in Pakistan. 